\title{
PRECISE - pregabalin in addition to usual care: statistical analysis plan
}

Stephanie Mathieson ${ }^{1 *}$, Laurent Billot ${ }^{1}$, Christopher G. Maher ${ }^{1}$, Andrew J. McLachlan², Jane Latimer ${ }^{1}$, Bart W. Koes ${ }^{3}$, Mark J. Hancock', Ian Harris ${ }^{5}$, Richard O. Day ${ }^{6}$, Justin Pik', Stephen Jan ${ }^{1}$ and Chung-Wei Christine Lin ${ }^{1}$

\begin{abstract}
Background: Sciatica is a severe, disabling condition that lacks high quality evidence for effective treatment strategies. This a priori statistical analysis plan describes the methodology of analysis for the PRECISE study.
\end{abstract}

Methods/design: PRECISE is a prospectively registered, double blind, randomised placebo controlled trial of pregabalin compared to placebo, in addition to usual care in patients with sciatica. The aim of this study is to determine the efficacy and cost-effectiveness of pregabalin in reducing leg pain intensity (primary outcome). Secondary outcomes include disability (key secondary), back pain intensity, quality of life, participants' perceived global effect, work absenteeism and health utilisation. Information about medication usage and tolerability are also collected. Outcomes are collected over one year (weeks 2, 4, 8, 12, 26 and 52). Double data entry will be conducted for primary and key secondary outcomes. Other outcomes will be checked using a risk-based approach. Analyses will be consistent with the intention-to-treat principle. Statistical tests will be two-tailed with a $p$ value $<0.05$ considered significant. Group allocation will remain masked until analyses and interpretation are finalised. Repeated-measure linear mixed models will assess the effect of treatment (pregabalin versus placebo) on primary and secondary outcomes at all time points. Fixed effects will include group allocation, visit as a categorical variable and the interaction between group and visit. Covariates will include baseline leg pain and symptom duration, with an interaction term between baseline leg pain and visit. Pairwise differences between groups will be tested at weeks 8 and 52 . The number of serious adverse events and adverse events will be reported, and the proportion of patients per group who have at least one event will be compared using Fisher's exact test. An economic evaluation will be conducted if there is a treatment effect on the primary outcome at week 8 . A subgroup analysis will assess whether presenting features of neuropathic pain at baseline modify the treatment effect of leg pain at week 8.

Discussion: This statistical analysis plan provides detailed methodology for the analysis of the PRECISE study, which aims to deliver much needed evidence about effective and affordable management of sciatica.

Trial registration: Australian and New Zealand Clinical Trials Registry (ACTRN12613000530729. Registered 13 May 2013)

Keywords: Sciatica, Pregabalin, Statistical analysis plan, Randomised controlled trial, Placebo

\footnotetext{
* Correspondence: smathieson@georgeinstitute.org.au

${ }^{1}$ The George Institute for Global Health and Sydney Medical School,

University of Sydney, P.O. Box M201Missenden Road, Sydney, NSW 2050,

Australia

Full list of author information is available at the end of the article
} 


\section{Update}

Sciatica is a severe, disabling condition characterised by radiating leg pain, with or without low back pain. There is limited high quality evidence for effective, conservative treatment strategies for patients with sciatica [1]. Pregabalin is a neuropathic pain medicine that may reduce leg pain in these patients; however, its use in this population is not informed by high quality direct evidence.

PRECISE is a randomised placebo-controlled trial evaluating the efficacy of pregabalin, in patients with sciatica, in addition to usual care [2]. Participant recruitment commenced in September 2013 and completed in March 2015. Data collection will be completed in April 2016. This statistical analysis plan details the planned analyses for the PRECISE study to facilitate transparency of data analysis. The statistical analysis plan was approved and signed by study investigators on 28 November 2015. Statistical analysis will be performed following data integrity checks and locking (estimated April 2016).

\section{Study overview Trial design}

PRECISE is a double-blind, randomised placebocontrolled trial of pregabalin compared to placebo, in addition to usual care, investigating the efficacy and cost-effectiveness of pregabalin in patients with sciatica. The study received ethics approval from the University of Sydney Human Research Ethics Committee (protocol number 15333), was prospectively registered at the Australian and New Zealand Clinical Trials Registry (ACTRN12613000530729) and the study protocol has been published [2].

\section{Study population}

Two hundred and nine eligible consenting participants who were seeking care for their back-related leg pain (sciatica) were recruited from primary care $(n=45$ sites) or outpatient specialist clinics $(n=2$ sites). Eligible participants were adults with radiating leg pain below the knee, of at least one week but no longer than one year in duration, which caused at least moderate pain or interference with work or daily activities in the last week. Participants had to have signs or symptoms of nerve root or spinal nerve involvement, as demonstrated by either myotomal weakness, dermatomal deficits, diminished reflexes or leg pain radiating in a dermatomal distribution, and had to have sufficient understanding of the English language or assistance with interpretation to allow completion of the study treatment and assessments. Participants were excluded if they had any known or suspected serious spinal pathology; were scheduled for spinal surgery or interventional procedures for sciatica during the 8-week treatment period; were pregnant, breastfeeding or planning conception during the intervention period; had taken pregabalin or gabapentin for this current episode of sciatica; were taking an anticonvulsant medication, a neuropathic pain medication, a tricyclic antidepressant or a sedative and unable to cease the medication; were suffering from severe depression or suicidal thoughts (score of $\geq 20$ on the Patient Health Questionnaire (PHQ-9) or a score of 2 or 3 on question 9); or had a contraindication(s) to pregabalin.

\section{Intervention}

All participants received advice (reassurance, advice to stay active and avoid prolonged bed rest) and a study medication pack containing either pregabalin or placebo. Participants commenced by taking one $75 \mathrm{mg}$ capsule, twice daily. Follow-up consultations (once a week for up to 8 weeks) with a study doctor monitored individual progress, tolerability and adverse events to titrate the dosage to the participant's optimal dose, up to a maximum $600 \mathrm{mg}$ per day. 'Adequate improvement' was defined as a pain rating of 0 or 1 out of 10 , for leg pain, for a minimum of 72 hours, with no or tolerable side effects. If adequate improvement was achieved before the maximum 8 week regimen was completed, early titration down to cessation was possible. In addition, usual care could be provided by the study doctor to all participants during the study and could include a referral for physical or manual therapy and/or prescription of analgesic medication. It was recommended that the study doctors follow the World Health Organisation (WHO) pain ladder [3] for analgesic medication prescription, and refrain from prescribing additional medicines for neuropathic pain (for example, antidepressants, selective serotonin and noradrenaline reuptake inhibitors, gabapentin and other anticonvulsant medications) or scheduling interventional procedures. The maximum treatment period was 8 weeks.

\section{Randomisation and allocation}

Study medicines were packaged and labelled according to a pre-generated random number sequence; each sealed box had a unique participant number. Allocation to either group (pregabalin or placebo) was at a 1:1 ratio. All study medicines were identical in appearance. The study doctor provided a prepackaged study medication pack to each eligible participant. All study personnel, study clinicians and participants were blind to the group allocation. A participant was considered randomised into the study once informed consent and baseline data were obtained and the participant was instructed to break the seal on the study medicine. 


\section{Outcome variables}

Outcomes were collected at baseline and at weeks 2, 4, $8,12,26$ and 52 (unless otherwise stated). Data were collected either by telephone, where a research assistant first entered the data on paper (source data) and then transcribed the data into the study electronic database, or by participants completing the study questionnaires online, with the data directly entered into the secure electronic database. Manual data checking procedures ensured correctness of data transfer of source data.

The primary outcome was leg pain intensity, measured by a Numeric Pain Rating Scale (NPRS). The NPRS asks participants to rate their leg pain on a scale of 10 , with 0 being 'no leg pain' and 10 being the 'worst pain imaginable', as an average over the last 24 hours [4]. The primary time point was week 8 .

Secondary outcomes were:

- Disability, measured using the Roland-Morris Disability Questionnaire for Sciatica [5]. It was the key secondary outcome.

- Back pain intensity, measured using the NPRS [4].

- Quality of life, measured using the Short Form Health Survey 12, version 2 (SF-12v2) questionnaire [6].

- Global Perceived Effect asks the participant to compare their leg pain to that experienced when commencing the trial and was measured on a Likert scale, from -5 vastly worse, to 0 unchanged, to +5 completely recovered [7].

- Work absenteeism, self-reported number of hours missed from paid employment due to leg pain (collected by self-report at weeks 4, 12, 26 and 52). Use of health services such as physiotherapy and use of medication for leg pain (other than the study medicine) (collected by self-report at weeks 4, 12, 26 and 52).

Additional data collected:

- Physical examination findings and level of nerve root involvement: at screening, the study doctor was asked to assess lower limb sensory, motor and reflex functions and provide their diagnosis on the level of the nerve root involved.

- Participant details: age, gender, self-reported height and weight.

- Socio-demographic details: health insurance status, employment status and household income.

- Episode details: leg pain duration, compensation status and medicine(s) use for leg pain one week preceding enrollment.

- Presence of neuropathic pain: evaluated at baseline using the painDETECT questionnaire [8].
- Details of serious adverse events and adverse events were collected, as well as confirmed pregnancy for both female and male (that is, partner pregnancy) participants up to week 12. Serious adverse events were defined as an event that was life threatening, resulted in death, hospitalisation, or significant disability. Adverse events were defined as any untoward medical occurrence as reported by the participant that may or may not be related to the study treatment.

- Study medication: study doctors were asked to record the prescribed study medication dosage for each participant weekly until medication cessation. Participants were asked to complete a diary, recording the daily consumption of study medication until cessation and to return any unused medicines at the end of week 8 . All returned medicines were counted and documented before secure destruction.

- Treatment satisfaction: participants were asked to rate, on a 5-point scale (extremely dissatisfied, dissatisfied, neutral, satisfied or extremely satisfied) how satisfied they were overall with the study treatment at week 8 .

- Participant blinding to treatment: participants were asked to guess to which study treatment they were randomised (pregabalin, placebo or don't know) at week 8 .

\section{Power and sample size}

A required sample size of 204 participants (102 per group) was calculated with $90 \%$ power to detect a difference of 1.5 units of leg pain out of the 10-unit NPRS at week 8. This assumed a standard deviation of 2.5 [9] and a two-tailed alpha of 0.05 , and allowed for $10 \%$ of dropouts and $20 \%$ non-compliance. The between-group difference of 1.5 units out of 10 in leg pain was based on previous trials of pregabalin for neuropathic pain conditions [10]. The sample size also had $90 \%$ power to detect a difference between groups of 3 on the 23-point Roland-Morris Disability Questionnaire for Sciatica, the key secondary outcome, at week 8 . This was based on the between-group difference of 3 and standard deviation of 4 from one of our previous sciatica trials [9], and the same assumptions taken for the primary outcome calculation.

\section{Statistical analysis Analysis principles}

Two analysts, blind to group allocation, will conduct independent analyses of the primary and key secondary outcomes. Results will be compared and discrepancies resolved. Other analyses will be conducted by one analyst with the codes reviewed by a senior statistician. A 
dummy dataset will be used to confirm statistical procedures, with discrepancies resolved before unblinding. Analyses will be consistent with the intention-to-treat principle. Participants will be analysed according to group allocation, estimating the mean difference at week 8 between pregabalin and placebo treatment groups regardless of the treatments received after randomisation. All statistical tests will be two-tailed and a $p$ value of $<0.05$ considered significant. Continuous variables will be summarised using standard measures of central tendency and dispersion, either as mean and standard error, or median and interquartile range. Dichotomous or categorical variables will be summarised by frequencies or denominators and percentages. Percentages will be calculated using the number of participants for whom data is available as the denominator. Analyses will be conducted using SAS software version 9.3 or above (SAS Institute Inc. 2012). No interim analysis will be conducted or has been planned, as pregabalin is used under its approved label use.

\section{Data integrity}

Integrity of trial data is monitored regularly by scrutinising data files for omissions and errors. Double data entry will be conducted for the primary (leg pain intensity) and key secondary (disability) outcomes. Other outcomes (that is, secondary outcomes excluding the key secondary) will be checked using a risk-based approach. This approach will be used for data collected by telephone, in which a random $10 \%$ sample of participants' paper files (source data) will be cross-checked against the electronic database. If the rate of error is greater than $10 \%$, another $10 \%$ sample is drawn and checked. The acceptable error rate for this new sample is then $9 \%$. If the error rate is again higher than the acceptable rate (9\%), then another $10 \%$ sample is drawn for checking and the next acceptable error rate is reduced by another $1 \%$. This process continues until the observed error rate is below the acceptable threshold. All inconsistencies will be investigated and rectified. For participants who directly completed their questionnaire(s) online, no cross-checking is required. Range checks will be performed on all variables.

\section{Blinding}

All researchers involved in the preparation of the analysis plan had no access to trial data broken down by treatment allocation. Once data quality checks are satisfactory and the database is locked, we will undertake a blind review to quantify missing data of the

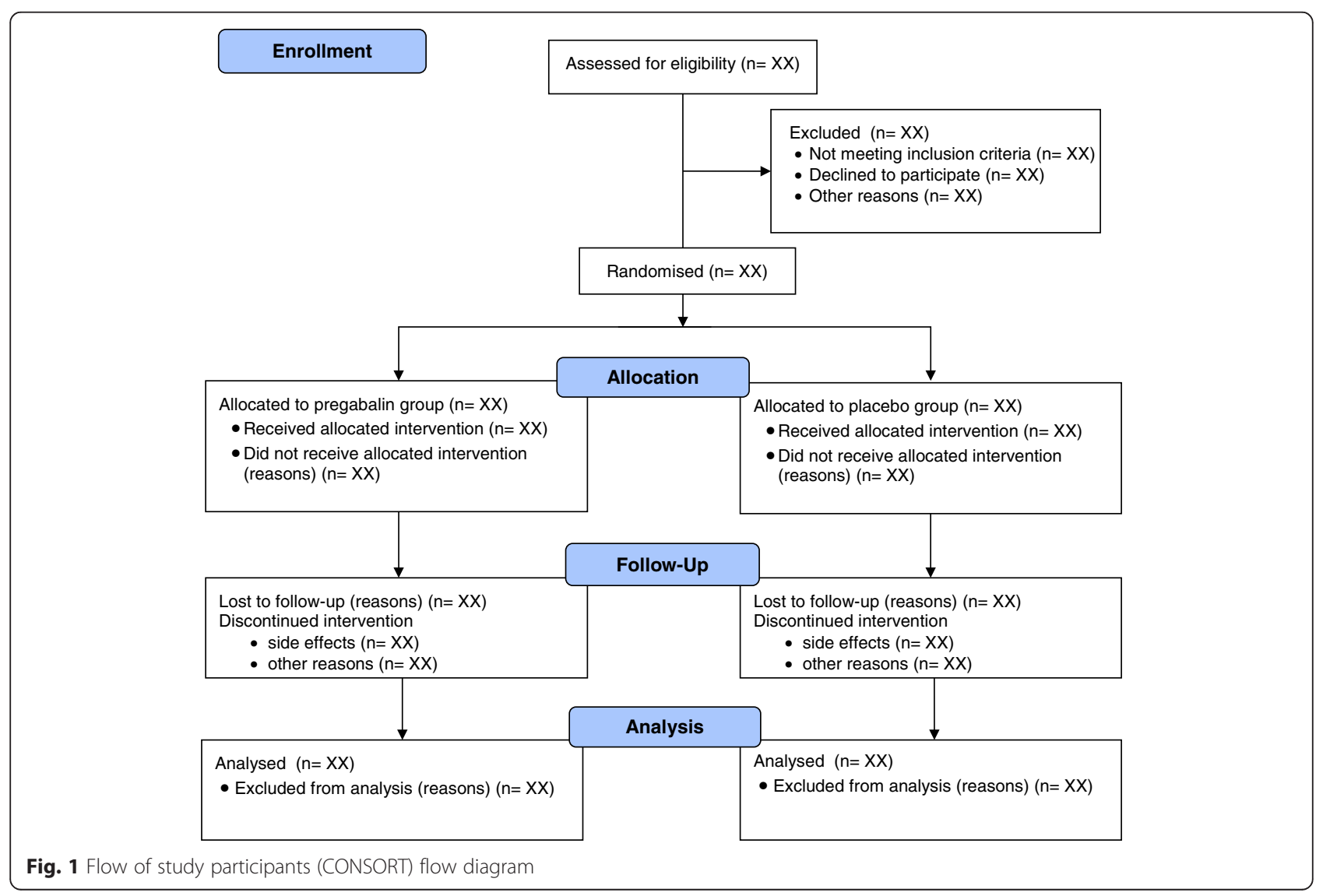


Table 1 Participant baseline characteristics

\begin{tabular}{|c|c|c|}
\hline & Pregabalin $(n=x x x)$ & Placebo $(n=x x x)$ \\
\hline \multicolumn{3}{|l|}{ Participant characteristics } \\
\hline Female & n/N (\%) & n/N (\%) \\
\hline Age (years) & $x x . x(x x . x$ to $x x . x), n$ & $x X . x(x X . x$ to $x X . x), n$ \\
\hline $\mathrm{BMI}\left(\mathrm{kg} / \mathrm{m}^{2}\right)$ & $x x . x(x x . x$ to $x x . x), n$ & $x x . x(x x . x$ to $x x . x), n$ \\
\hline Currently employed & n/N (\%) & n/N (\%) \\
\hline Compensable leg pain & n/N (\%) & n/N (\%) \\
\hline \multicolumn{3}{|l|}{ Household income/week (year) (AUD) } \\
\hline No income & n/N (\%) & n/N (\%) \\
\hline$\$ 1-\$ 649(\$ 1-\$ 33,799)$ & n/N (\%) & $\mathrm{n} / \mathrm{N}(\%)$ \\
\hline$\$ 650-\$ 1699(\$ 33,800-\$ 88,399)$ & n/N (\%) & n/N (\%) \\
\hline$\$ 1700-\$ 3999(\$ 88,400-\$ 207,999)$ & $\mathrm{n} / \mathrm{N}(\%)$ & n/N (\%) \\
\hline$\$ 4000$ or more ( $\$ 208,000$ or more) & n/N (\%) & n/N (\%) \\
\hline Chose not to answer & $\mathrm{n} / \mathrm{N}(\%)$ & n/N (\%) \\
\hline \multicolumn{3}{|l|}{ Medicine use in the last week ${ }^{a}$} \\
\hline Simple analgesics & n/N (\%) & n/N (\%) \\
\hline NSAIDs & $\mathrm{n} / \mathrm{N}(\%)$ & $\mathrm{n} / \mathrm{N}(\%)$ \\
\hline Strong opioid analgesics & n/N (\%) & n/N (\%) \\
\hline Combination opioid analgesics & n/N (\%) & n/N (\%) \\
\hline Other & $\mathrm{n} / \mathrm{N}(\%)$ & n/N (\%) \\
\hline \multicolumn{3}{|l|}{ Occupation } \\
\hline Manager & n/N (\%) & n/N (\%) \\
\hline Technician and Trade Worker & n/N (\%) & n/N (\%) \\
\hline Clerical and Administrative Worker & n/N (\%) & $\mathrm{n} / \mathrm{N}(\%)$ \\
\hline Machinery Operator and Driver & n/N (\%) & n/N (\%) \\
\hline Professional & $\mathrm{n} / \mathrm{N}(\%)$ & n/N (\%) \\
\hline Community and Personal Service Worker & n/N (\%) & n/N (\%) \\
\hline Sales Worker & n/N (\%) & n/N (\%) \\
\hline Labourer & n/N (\%) & n/N (\%) \\
\hline \multicolumn{3}{|l|}{ Health insurance } \\
\hline None & n/N (\%) & n/N (\%) \\
\hline Private hospital only & n/N (\%) & n/N (\%) \\
\hline Private ancillary (extras) only & n/N (\%) & n/N (\%) \\
\hline Private hospital and ancillary (extras) & $\mathrm{n} / \mathrm{N}(\%)$ & n/N (\%) \\
\hline DVA & n/N (\%) & n/N (\%) \\
\hline Chose not to answer & n/N (\%) & n/N (\%) \\
\hline \multicolumn{3}{|l|}{ Episode characteristics } \\
\hline \multicolumn{3}{|l|}{ Physical examination } \\
\hline Dermatomal pain & n/N (\%) & n/N (\%) \\
\hline Neurological deficit & n/N (\%) & n/N (\%) \\
\hline Sensory deficit & n/N (\%) & $\mathrm{n} / \mathrm{N}(\%)$ \\
\hline Motor deficit & n/N (\%) & n/N (\%) \\
\hline \multicolumn{3}{|l|}{ Clinician diagnosis } \\
\hline Spinal level affected $-\llcorner 3$ & n/N (\%) & n/N (\%) \\
\hline Spinal level affected $-\llcorner 4$ & n/N (\%) & n/N (\%) \\
\hline
\end{tabular}


Table 1 Participant baseline characteristics (Continued)

\begin{tabular}{|c|c|c|}
\hline Spinal level affected $-\mathrm{L} 5$ & $\mathrm{n} / \mathrm{N}(\%)$ & $\mathrm{n} / \mathrm{N}(\%)$ \\
\hline Spinal level affected -S1 & $\mathrm{n} / \mathrm{N}(\%)$ & $\mathrm{n} / \mathrm{N}(\%)$ \\
\hline Spinal level affected -\$2 & $\mathrm{n} / \mathrm{N}(\%)$ & $\mathrm{n} / \mathrm{N}(\%)$ \\
\hline Multiple spinal levels affected & $\mathrm{n} / \mathrm{N}(\%)$ & $\mathrm{n} / \mathrm{N}(\%)$ \\
\hline Not reported & $\mathrm{n} / \mathrm{N}(\%)$ & $\mathrm{n} / \mathrm{N}(\%)$ \\
\hline Leg pain duration (days) & $x x . x(x x . x$ to $x x . x), n$ & $x x . x(x X . x$ to $x x . x), n$ \\
\hline Leg pain intensity (NPRS) & $x X . x(x X . x$ to $x X . x), n$ & $X X . X(X X . X$ to $X X . X), n$ \\
\hline Back pain intensity (NPRS) & $X X . X(X X . X$ to $X X . X), n$ & $X X . X(x X . X$ to $X X . X), n$ \\
\hline Disability (Roland Disability Questionnaire Sciatica) & $x x . x(x x . x$ to $x x . x), n$ & $x x . x(x x . x$ to $x x . x), n$ \\
\hline Quality of life-physical score (SF-12v2) & XX.X (XX.X to XX.X), n & $x x . x(x x . x$ to $x x . x), n$ \\
\hline Quality of life-mental score (SF-12v2) & $X X . X(x X . X$ to $X X . X), n$ & $X X . X(X X . X$ to $X X . X), n$ \\
\hline Global Perceived Effect scale & $x x . x(x x . x$ to $x x . x), n$ & $x x . x(x x . x$ to $x x . x), n$ \\
\hline \multicolumn{3}{|l|}{ painDETECT score } \\
\hline score $\leq 12$ (neuropathic component unlikely) & $\mathrm{n} / \mathrm{N}(\%)$ & $\mathrm{n} / \mathrm{N}(\%)$ \\
\hline score 13-18 (unclear if neuropathic pain present) & $\mathrm{n} / \mathrm{N}(\%)$ & $\mathrm{n} / \mathrm{N}(\%)$ \\
\hline score $\geq 19$ (neuropathic component likely) & n/N (\%) & n/N (\%) \\
\hline
\end{tabular}

Abbreviations: DVA Department of Veterans' Affair, NPRS Numeric Pain Rating Scale, SF-12v2 Short Form Health Survey 12, version 2

aExamples of medicines include simple analgesics such as paracetamol, NSAIDs such as ibuprofen, strong opioid analgesic such as oxycodone and combination opioid analgesics such as paracetamol/codeine preparations

entire dataset (that is, not separated by group) and allow for any final amendments to the statistical analysis plan. During analysis and interpretation, group allocation will be masked using dummy group names (for example, group A, group B). The true group allocation will be unmasked only after the final statistical report has been completed and interpretation agreed upon.

\section{Methods for handling missing data}

In case more than $10 \%$ of the primary outcome data (leg pain intensity) at week 8 is missing, multiple imputations will be used to conduct sensitivity analyses for the longitudinal linear mixed model of the primary outcome. Ten imputations using chained equations will be used to replace missing leg pain data across all visits. In addition to the outcome itself, the imputation model will include a range of prognostic baseline variables. The need to impute as well as the list of prognostic variables for the multiple imputations will be confirmed at the time of the blind review.

\section{Trial profile}

The flow of participants through the study will be reported and will comply with the Consolidated Standards of Reporting Trials (CONSORT) (Fig. 1).

\section{Evaluation of demographics and baseline characteristics} Descriptive statistics of the baseline characteristics will be presented by treatment group (Table 1) and will include participant and episode characteristics.

\section{Primary analyses}

Repeated-measure linear mixed models will be used to assess the effect of treatment (pregabalin versus placebo) on leg pain intensity at all time points (weeks 2, 4, 8, 12, 26 and 52). Fixed effects will include group allocation, visit as a categorical variable and the interaction between group and visit. In addition, baseline leg pain (NPRS score) and symptom duration (days of symptom duration at baseline) will be included as covariates (Table 2) with an interaction term between baseline leg pain and visit. For every time point, we will calculate the adjusted mean leg pain score (NPRS score) per group as well as corresponding $95 \%$ confidence intervals. We will formally test adjusted mean differences between groups at the week 8 and week 52 time points (Table 2) with week 8 as the primary comparison. We will consider an effect size to be clinically significant if there is a between-group difference of 1.5 units of leg pain out of 10 units on the NPRS. Within patient correlations will be modelled using a repeated effect and a compound symmetry covariance structure. As sensitivity analyses, the model will also be run using a heterogeneous compound symmetry structure and a spatial power structure.

\section{Secondary analyses}

Secondary outcomes, with the exception of work absenteeism, health service utilisation and medicine use, will be analysed using the same method as the primary analysis (longitudinal linear models) to evaluate the effect of 
Table 2 Results of longitudinal linear mixed model and Fisher exact test for primary and secondary outcomes (in mean and SE for continuous data, number and percentage for count data)

\begin{tabular}{|c|c|c|c|}
\hline & \multicolumn{2}{|l|}{ Adjusted mean (SE) } & \multirow[b]{2}{*}{ Mean difference $(95 \% \mathrm{Cl})\{p$ value $\}$} \\
\hline & Pregabalin $(n=x x x)$ & Placebo $(n=x x x)$ & \\
\hline \multicolumn{4}{|c|}{ Leg pain intensity (NPRS) } \\
\hline Week 2 & $x x . x(x x . x$ to $x x . x), n$ & $x x . x(x x . x$ to $x x . x), n$ & \\
\hline Week 4 & $x x . x(x x . x$ to $x x . x), n$ & $x x . x(x x . x$ to $x x . x), n$ & \\
\hline Week 8 & $x x . x(x x . x$ to $x x . x), n$ & $x x . x(x x . x$ to $x x . x), n$ & $x x . x(x x . x$ to $x x . x)\{p$ value $\}$ \\
\hline Week 12 & $x x . x(x x . x$ to $x x . x), n$ & $x x . x(x x . x$ to $x x . x), n$ & \\
\hline Week 26 & $x x . x(x X . x$ to $x x . x), n$ & $x x . x(x x . x$ to $x x . x), n$ & \\
\hline Week 52 & $x x \cdot x(x x . x$ to $x x . x), n$ & $x x . x(x x . x$ to $x x . x), n$ & Xx.x (xx.x to Xx.x) \{p value\} \\
\hline \multicolumn{4}{|c|}{ Disability (RDQS) } \\
\hline Week 2 & $x x . x(x X . x$ to $x X . X), n$ & $x x . x(x X . x$ to $x X . x), n$ & \\
\hline Week 4 & $x x . x(x x . x$ to $x x . x), n$ & $x x . x(x x . x$ to $x x . x), n$ & \\
\hline Week 8 & $x x . x(x x . x$ to $x x . x), n$ & $x x . x(x X . x$ to $x X . x), n$ & xx.x (xx.x to xx.x) \{p value\} \\
\hline Week 12 & $x x . x(x x . x$ to $x x . x), n$ & $x x . x(x x . x$ to $x x . x), n$ & \\
\hline Week 26 & $x X . x(x X . x$ to $x X . x), n$ & $x x . x(x X . x$ to $x x . x), n$ & \\
\hline Week 52 & $x x . x(x x . x$ to $x x . x), n$ & $x x . x(x x . x$ to $x x . x), n$ & $x x . x(x x . x$ to $x x . x)\{p$ value $\}$ \\
\hline \multicolumn{4}{|c|}{ Back pain intensity (NPRS) } \\
\hline Week 2 & $x x . x(x x . x$ to $x x . x), n$ & $x x . x(x x . x$ to $x x . x), n$ & \\
\hline Week 4 & $x x . x(x x . x$ to $x x . x), n$ & $x x . x(x x . x$ to $x x . x), n$ & \\
\hline Week 8 & $x x . x(x x . x$ to $x x . x), n$ & $x x . x(x x . x$ to $x x . x), n$ & xx.x (xx.x to xx.x) \{p value\} \\
\hline Week 12 & $x x . x(x x . x$ to $x x . x), n$ & $x x . x(x x . x$ to $x x . x), n$ & \\
\hline Week 26 & $x x . x(x x . x$ to $x x . x), n$ & $x x . x(x x . x$ to $x x . x), n$ & \\
\hline Week 52 & $x x . x(x x . x$ to $x x . x), n$ & $x x . x(x x . x$ to $x x . x), n$ & xx.x (xx.x to xx.x) \{p value\} \\
\hline \multicolumn{4}{|c|}{ Global Perceived Effect } \\
\hline Week 2 & $x x . x(x x . x$ to $x x . x), n$ & $x x . x(x x . x$ to $x x . x), n$ & \\
\hline Week 4 & $x x . x(x X . x$ to $x x . x), n$ & $x X . X(X X . x$ to $x X . X), n$ & \\
\hline Week 8 & $x x . x(x x . x$ to $x x . x), n$ & $x X . x(x X . x$ to $x X . X), n$ & Xx.x (xx.x to Xx.x) \{p value\} \\
\hline Week 12 & $x x . x(x x . x$ to $x x . x), n$ & $x x . x(x x . x$ to $x x . x), n$ & \\
\hline Week 26 & $x X . x(x X . x$ to $x X . x), n$ & $x X . x(x X . x$ to $x X . x), n$ & \\
\hline Week 52 & $x x . x(x x . x$ to $x x . x), n$ & $x x . x(x x . x$ to $x x . x), n$ & xx.x (xx.x to xx.x) \{p value\} \\
\hline \multicolumn{4}{|c|}{ Quality of life - physical score (SF12-v2) } \\
\hline Week 2 & $x x . x(x x . x$ to $x x . x), n$ & $x x . x(x x . x$ to $x x . x), n$ & \\
\hline Week 4 & $x x . x(x x . x$ to $x x . x), n$ & $x x . x(x x . x$ to $x x . x), n$ & \\
\hline Week 8 & $x x . x(x X . x$ to $x x . x), n$ & $x x . x(x x . x$ to $x x . x), n$ & Xx.x (xx.x to xx.x) \{p value\} \\
\hline Week 12 & $x x . x(x x . x$ to $x x . x), n$ & $x x . x(x x . x$ to $x x . x), n$ & \\
\hline Week 26 & $x X . x(x X . x$ to $x X . X), n$ & $x x . x(x X . x$ to $x x . x), n$ & \\
\hline Week 52 & $x x . x(x X . x$ to $x x . x), n$ & $x x . x(x x . x$ to $x x . x), n$ & xx.x (xx.x to xx.x) \{p value\} \\
\hline \multicolumn{4}{|c|}{ Quality of life - mental score (SF12-v2) } \\
\hline Week 2 & $x x . x(x x . x$ to $x x . x), n$ & $x x . x(x x . x$ to $x x . x), n$ & \\
\hline Week 4 & $x X . x(x X . x$ to $x X . x), n$ & $x X . x(x X . x$ to $x X . x), n$ & \\
\hline Week 8 & $x x . x(x x . x$ to $x x . x), n$ & $x x . x(x x . x$ to $x x . x), n$ & Xx.x (xx.x to xx.x) \{p value\} \\
\hline Week 12 & $x x . x(x x . x$ to $x x . x), n$ & $x x . x(x x . x$ to $x x . x), n$ & \\
\hline Week 26 & $x x . x(x x . x$ to $x x . x), n$ & $x x . x(x x . x$ to $x x . x), n$ & \\
\hline Week 52 & $x x . x(x x \cdot x$ to $x x . x), n$ & $x x . x(x x . x$ to $x x . x), n$ & $x x . x(x x . x$ to $x x . x)\{p$ value $\}$ \\
\hline
\end{tabular}


Table 2 Results of longitudinal linear mixed model and Fisher exact test for primary and secondary outcomes (in mean and SE for continuous data, number and percentage for count data) (Continued)

\begin{tabular}{|c|c|c|c|}
\hline \multicolumn{4}{|l|}{ Work absenteeism } \\
\hline Hours absent from work & $x x x . x(x x x . x$ to $x x x . x), n$ & $x x x . x(x x x . x$ to $x x x . x), n$ & xxx.x (xxx.x to xxx.x) \{p value\} \\
\hline \multicolumn{4}{|l|}{ Use of other treatments } \\
\hline Participants using other medicines $\{$ Fisher test\} & $\mathrm{n} / \mathrm{N}(\%)$ & $\mathrm{n} / \mathrm{N}(\%)$ & $x x . x(x x . x$ to $x x . x)\{p$ value $\}$ \\
\hline Participants using health services & $\mathrm{n} / \mathrm{N}(\%)$ & $\mathrm{n} / \mathrm{N}(\%)$ & $x x . x(x x . x$ to $x x . x)\{p$ value $\}$ \\
\hline
\end{tabular}

Abbreviations: SE standard error, NPRS Numeric Pain Rating Scale, RDQS Roland-Morris Disability Questionnaire Sciatica

Model terms include visit (categorical), treatment by visit interaction, baseline leg pain, baseline symptom duration and baseline leg pain by visit interaction

the intervention over one year (Table 2). We will test pairwise differences between groups for secondary outcomes at the week 8 and week 52 time points (Table 2). We will consider an effect size to be clinically significant if there is a between-group difference of 1.5 units out of 10 units on the NPRS for back pain [10], and 3 points on the 23-point scale Roland-Morris Disability Questionnaire for Sciatica for disability [9]. Work absenteeism and health services utilisation will be calculated as the cumulative number between baseline and week 52, analysed using analysis of covariance (ANCOVA), adjusted for symptom duration. Use of medicine(s) (that is, cointervention) will be calculated as the percentage of participants reporting to be taking at least one medicine (other than the study medicine) for their leg pain and differences compared between groups using the Fisher exact test.

\section{Evaluation of serious adverse events and adverse events} All participants reporting serious adverse events were systematically investigated at the time of being reported for potential association with the study treatment. Serious adverse events and adverse events will be coded into categories according to the World Health Organisation's International Classification of Diseases (ICD-10) using three-digit codes [11]. For each category, we will report the number of events and compare the proportion of patients with at least one event between groups using Fisher's exact test (Table 3).

\section{Evaluation of adherence to the study treatment}

Adherence to the study treatment will be defined as having consumed $\geq 80 \%$ of the study medicine (measured by self-report in the medication diary) against the study doctor's prescription [12]. This will be supported by the participant's returned medication count (Table 4).

\section{Evaluation of participant blinding and satisfaction to treatment}

The number of participants reporting to which group they thought they were allocated and the number of participants reporting their level of satisfaction with the study treatment will be reported per group (Table 4).

\section{Economic evaluation}

Economic evaluation will be conducted if there is a treatment effect on the primary outcome at week 8 . It would entail a cost-utility analysis in which the intervention will be assessed in terms of its incremental cost per quality-adjusted life year (QALY). As we do not expect any effect on survival, our QALY estimates will be based exclusively on health state utilities. These will be obtained from measures derived from the SF-12 and transformed into health state utilities via the SF-6D algorithm [13]. For each participant, these utilities will be averaged out between observations over the entire duration of follow-up of one year. The primary analysis will be conducted from the perspective of the health sector. Healthcare services and medicines will be valued using

Table 3 Serious adverse events and adverse events reported. Events grouped using International Classification of Diseases (ICD10) categories

\begin{tabular}{lll}
\hline & Pregabalin $(n=x x)$ & Placebo $(n=x x x)$ \\
\hline $\begin{array}{l}\text { Serious adverse events } \\
\text { Total \{Fisher test\} }\end{array}$ & $\mathrm{n}_{\text {evt }} \mathrm{n}_{\text {pat }}(\%)$ & $\mathrm{n}_{\text {evt }} \mathrm{n}_{\text {pat }}(\%)$ \\
Adverse events & & \\
Total \{Fisher test\} & $\mathrm{n}_{\text {evt }} \mathrm{n}_{\text {pat }}(\%)$ & $\mathrm{n}_{\text {evt }} \mathrm{n}_{\text {pat }}(\%)$ \\
Adverse event A & $\mathrm{n}_{\text {evt }} \mathrm{n}_{\text {pat }}(\%)$ & $\mathrm{n}_{\text {evt }} \mathrm{n}_{\text {pat }}(\%)$ \\
Adverse event B & $\mathrm{n}_{\text {evt }} \mathrm{n}_{\text {pat }}(\%)$ & $\mathrm{n}_{\text {evt }} \mathrm{n}_{\text {pat }}(\%)$ \\
And so on & $\mathrm{n}_{\text {evt }} \mathrm{n}_{\text {pat }}(\%)$ & $\mathrm{n}_{\text {evt }} \mathrm{n}_{\text {pat }}(\%)$ \\
\hline
\end{tabular}


Table 4 Result of process variables (in mean and SE for continuous data, number and percentage for count data)

\begin{tabular}{|c|c|c|}
\hline & Pregabalin $(n=x x x)$ & Placebo $(n=x x x)$ \\
\hline \multicolumn{3}{|l|}{ Self-reported daily dose (mg/day) } \\
\hline Week 1 & $x x x . x(x x x . x$ to $x x x . x), n$ & $x x x . x(x x x . x$ to $x x x . x), n$ \\
\hline Week 2 & $x x x . x(x x x . x$ to $x x x . x), n$ & $x x x . x(x x x . x$ to $x x x . x), n$ \\
\hline Week 3 & $x x x . x(x x x . x$ to $x x x . x), n$ & $x x x . x(x x x . x$ to $x x x . x), n$ \\
\hline Week 4 & $x x x . x(x x x . x$ to $x x x . x), n$ & $x x x . x(x x x . x$ to $x x x . x), n$ \\
\hline Week 5 & $x x x . x(x x x . x$ to $x x x . x), n$ & $x x x . x(x x x . x$ to $x x x . x), n$ \\
\hline Week 6 & $x x x . x(x x x . x$ to $x x x . x), n$ & $x x x . x(x x x . x$ to $x x x . x), n$ \\
\hline Week 7 & $x x x . x(x x x . x$ to $x x x . x), n$ & $x x x . x(x x x . x$ to $x x x . x), n$ \\
\hline Week 8 & $x x x . x(x x x . x$ to $x x x . x), n$ & $x x x . x(x x x . x$ to $x x x . x), n$ \\
\hline Participants returning study medicines & n/N (\%) & n/N (\%) \\
\hline \multicolumn{3}{|c|}{ Participants consuming $\geq 80 \%$ prescribed dose } \\
\hline Medication diary & n/N (\%) & n/N (\%) \\
\hline Returned medicine count & n/N (\%) & n/N (\%) \\
\hline \multicolumn{3}{|l|}{ Assessment of participant blinding } \\
\hline Pregabalin & n/N (\%) & n/N (\%) \\
\hline Placebo & n/N (\%) & n/N (\%) \\
\hline Don't know & n/N (\%) & n/N (\%) \\
\hline \multicolumn{3}{|l|}{ Treatment satisfaction } \\
\hline Very dissatisfied & n/N (\%) & n/N (\%) \\
\hline Dissatisfied & n/N (\%) & n/N (\%) \\
\hline Neutral & n/N (\%) & n/N (\%) \\
\hline Satisfied & n/N (\%) & n/N (\%) \\
\hline Very satisfied & n/N (\%) & n/N (\%) \\
\hline
\end{tabular}

published sources where possible. An additional analysis will entail a societal perspective, including costs associated work absenteeism because of sciatica. Costs of absenteeism from paid employment will be estimated by the number of days absent from work multiplied by the average wage rate. The incremental cost per QALY will be estimated as the ratio of the difference in average cost and QALYs between intervention arms. If required, a sensitivity analysis will test uncertainty in key parameters such as the selection of cost weights and statistical variation in quality of life scores.

\section{Subgroup analyses}

A planned subgroup analysis [2] will be conducted on the primary outcome assessing neuropathic pain features of participants at baseline to determine role of neuropathic pain as a modifier of treatment effects at week 8 . This will be conducted using linear mixed models with the addition of neuropathic pain and its interaction with the allocation group. We will use the recommended cut-off values of the painDETECT questionnaire [8] to categorise the presence of neuropathic pain: a score $\geq 19$ represents that a neuropathic component is likely ( $>90 \%$ ); a score $\leq 12$ represents that neuropathic pain is unlikely $(<15 \%)$; a score between
13-18 indicates that it is unclear if neuropathic pain is present.

\section{Conclusion}

The PRECISE study aims to provide much needed evidence about effective and affordable management of the debilitating symptoms of sciatica. This statistical analysis plan details the study's planned analyses, to aid transparency of results, and may assist the design of studies in the future.

\section{Trial status}

Participant recruitment was completed in late March 2015 and follow-up outcomes will be collected by early April 2016.

\footnotetext{
Abbreviations

ANCOVA: analysis of covariance; $\mathrm{Cl}$ : confidence interval; CONSORT: Consolidated Standards of Reporting Trials; NPRS: Numeric Pain Rating Scale; PHQ-9: Patient Health Questionnaire; QALY: quality-adjusted life year; SE: standard error; SF-12v2: Short Form Health Survey 12, version 2.

\section{Competing interests}

The study medications were provided by in-kind support from Pfizer Australia, but Investigators maintain full autonomy in the design, conduct, analyses and reporting of the study. There are no other competing interests.
} 


\section{Authors' contributions}

CWCL, AJM, JL, BWK, MH, ROD and CGM conceived the trial. CWCL, CGM, $A J M, J L, B W K, M H, I H, R O D, J P, S J$ and $L B$ procured trial funding. CWCL, AJM $J L, B W K, M H, R O D, C G M, I H, J P, S J, L B$ and SM contributed to the design of the trial. CWCL, CGM, AJM, JL, BWK, MH, IH and ROD formed the steering committee and end point adjudication. LB oversaw the design of the statistical analyses. CWCL, AJM, JL, BWK, MH, ROD, CGM, IH, JP, SJ, LB and SM were involved in the analysis planning. SM drafted the manuscript. CWCL, AJM, JL, BWK, MH, ROD, CGM, IH, JP, SJ, LB and SM contributed to the manuscript and approved the final version for publication.

\section{Authors' information}

SM, PhD student; LB, Director, Statistics Division and Principal Research Fellow; CGM, PhD, Director and Professor of Physiotherapy; JL, PhD, Professor of Physiotherapy, SJ, Professor, Senior Health Economist, and CWCL, PhD, Associate Professor are from The George Institute for Global Health and Sydney Medical School, The University of Sydney, Sydney, Australia. AJM, PhD, Professor of Pharmacy, The University of Sydney, Sydney, Australia. BWK, PhD, Professor, Department of General Practice, Erasmus University Medical Center, Rotterdam, The Netherlands. MH, PhD, Associate Professor, Faculty of Human Sciences, Macquarie University, Sydney, Australia. IH, Professor, The South Western Sydney Clinical School, Faculty of Medicine, University of New South Wales, Sydney, Australia. ROD, PhD, Professor, Department of Clinical Pharmacology \& Toxicology, University of New South Wales, Sydney, Australia. JP, Neurosurgeon, ACT NeuroSpine Clinic, Deakin, Australian Capital Territory, Australia.

\section{Acknowledgements}

PRECISE is an investigator-initiated trial funded by Australia's National Health and Medical Research Council (NHMRC) (ID APP1042073). Investigators maintain full autonomy in the design, conduct, analyses and reporting of the study. JL holds a Future Fellowship funded by the Australian Research Council. SJ and CGM hold NHMRC Senior Research Fellowships, CWCL holds an NHMRC Career Development Fellowship. SM holds a postgraduate scholarship from the NHMRC.

\section{Author details}

'The George Institute for Global Health and Sydney Medical School, University of Sydney, P.O. Box M201Missenden Road, Sydney, NSW 2050, Australia. ${ }^{2}$ Faculty of Pharmacy and Centre for Education and Research on Ageing, University of Sydney, Sydney, Australia. ${ }^{3}$ Department of General Practice, Erasmus University Medical Center, Rotterdam, The Netherlands. ${ }^{4}$ Faculty of Human Sciences, Macquarie University, Sydney, Australia. ${ }^{5}$ The South Western Sydney Clinical School, Faculty of Medicine, University of New South Wales, Sydney, Australia. ${ }^{6}$ St Vincent's Clinical School, Faculty of Medicine, University of New South Wales, Sydney, Australia. ${ }^{7}$ ACT NeuroSpine Clinic, Deakin, Australian Capital Territory, Australia.

Received: 10 August 2015 Accepted: 13 January 2016

Published online: 27 January 2016

\section{References}

1. Finnerup NB, Attal N, Haroutounian S, McNicol E, Baron R, Dworkin RH, et al. Pharmacotherapy for neuropathic pain in adults: a systematic review and meta-analysis. Lancet Neurol. 2015;14:162-73.

2. Mathieson S, Maher CG, McLachlan AJ, Latimer J, Koes BW, Hancock MJ, et al. PRECISE - pregabalin in addition to usual care for sciatica: study protocol for a randomised controlled trial. Trials. 2013:14:1-8.

3. Ehrlich GE. Low back pain. Bull World Health Organ. 2003;81:671-6.

4. Williamson A. Pain: a review of three commonly used pain rating scales. Issues Clin Nurs. 2004;14:798-804.

5. Patrick DL, Deyo RA, Atlas SJ, Singer DE, Chapin A, Keller RB. Assessing helathrelated quality of life in patients with sciatica. Spine. 1995;20:1899-909.

6. Ware JE, Kosinski M, Turner-Bowker DM, Gandek B. User's Manual for the SF-12v2 ${ }^{\circledR}$ Health Survey (With a Supplement Documenting SF-12 ${ }^{\circledR}$ Health Survey). Quality Metric Incorporated: Lincoln; 2002.

7. Kamper SJ, Ostelo RW, Knol DL, Maher CG, de Vet HC, Hancock MJ. Global Perceived Effect scales provided reliable assessments of health transition in people with musculoskeletal disorders, but ratings are strongly influenced by current status. J Clin Epidemiol. 2010;63:760-6.
8. Freynhagen R, Baron R, Gockel U, Tolle TR. painDETECT: a new screening questionnaire to identify neuropathic components in patients with back pain. Curr Med Res Opin. 2006;22:1911-20.

9. Peul WC, van Houwelingen $\mathrm{HC}$, van den Hout WB, Brand $\mathrm{R}$, Eekhof JAH Tans JT, et al. Surgery versus prolonged conservative treatment for sciatica. N Engl J Med. 2007;356:2245-56.

10. Dworkin RH, Turk DC, McDermott MP, Peirce-Sandner S, Burke LB, Cowan P, et al. Interpreting the clinical importance of group differences in chronic pain clinical trials: IMMPACT recommendations. Pain. 2009:146:238-44.

11. World Health Organization. International statistical classification of diseases and related health problems. vol 10th revision, edition. Geneva:WHO Library Cataloguing-in-Publication Data; 2010

12. Choudhry NK, Glynn RJ, Avorn J, Lee JL, Brennan TA, Reisman L, et al. Untangling the relationship between medication adherence and postmyocardial infarction outcomes: medication adherence and clinical outcomes. Am Heart J. 2014;167:51-8. e5.

13. Braizer JE, Roberts J. The estimation of a preference-based measure of health from the SF-12. Med Care. 2004:42:851-9.

\section{Submit your next manuscript to BioMed Central and we will help you at every step:}

- We accept pre-submission inquiries

- Our selector tool helps you to find the most relevant journal

- We provide round the clock customer support

- Convenient online submission

- Thorough peer review

- Inclusion in PubMed and all major indexing services

- Maximum visibility for your research

Submit your manuscript at www.biomedcentral.com/submit
Biomed Central 